how to divide - especially in the stage of their life cycle when they are in the bloodstream, causing the human and animal disease. Indeed, the flagellum seems to be the tail that wags the dog. These results raise the intriguing possibility of developing drugs that act selectively against the trypanosome-specific axonemal proteins. It may be possible to identify compounds that cripple the parasite flagellum while leaving the distinct proteins of the human axoneme alone.
ScottM. Landfear is in the Department of Molecular Microbiology and Immunology, Oregon Health and Science University, Portland, Oregon 97239 , USA.

e-mail:landfear@ohsu.edu

1. Broadhead, R.et al. Nature 440, 224-227(2006)

2. Pazour, G Let a.J. Cell Bial. $770,103-113(2005)$.

3. Smith, I C.etal. . Prateome Res. 4,909-919 (2005).

4. www.who.int/mediacentre/factsheets/4s299/en/ indexhtml

5. Moreira-Leite, F.F.et a. Sdence 294, 610-612(2001).

\title{
ASTROPHYSICS
}

\section{Ancient blast comes to light}

\author{
Enrico Ramirez-Ruiz
}

\section{Light from the oldest and farthest stellar explosion yet seen was emitted when the Universe was a mere infant. It provides a close-up view of how and when stars formed, and how they affect the primordial gas around them.}

A trio of contributions to this issue $\mathrm{e}^{1-3}$ presents observations of the most distant stellar explosion ever seen: a $\gamma$-ray burst (GRB) that took place when the Universe (currently accepted age, roughly 13.7 billion years) was only about 900 million years old. Thus, for the first time, the most distant objects that can be identified spectroscopically are not just galaxies - and therefore huge agglomerates of stars, gas and dust - but also individual stars.

The hunt for cosmic structures that ignited when the Universe was in its infancy takes us to the frontier of our current observational capabilities (Fig. 1). Not too long ago, the most distant objects known were quasi-stellar objects, or quasars ${ }^{4}$ - glaringly luminous objects powered by gas falling into massive black holes at the centres of galaxies. Over the past few years, however, extremely sensitive surveys with space- and ground-based telescopes have allowed us to observe ordinary galaxies beyond the farthest quasars ${ }^{5}$.

Since then, the race has been on to find the most distant star. As most stars lead relatively unexciting lives, they remain far less luminous than galaxies, and so distant stars are generally too faint to be detected with current technology. Some heavyweight stars, however, end their lives violently and spectacularly. These stars send out bursts of radiation so luminous that they appear bright even when viewed across vast stretches of the Universe.

On 4 September 2005 , such a flash of $\gamma$-rays, lasting for 80 seconds, hit NASA's purposebuilt GRB-detection satellite, Swift. Swift's $\gamma$-ray monitor established the position of the burst - prosaically labelled GRB 050904 - in the constellation Pisces. As Cusumano et al. describe (page 164) ${ }^{1}$, within seconds Swift turned around to direct its X-ray telescope at the region where the burst occurred, pinpointing the location of a rapidly fading source of radiation to within a hundredth of a degree. This in turn allowed powerful optical and infrared telescopes around the world to search for the decaying 'afterglow' signal within their wavelength ranges. On page 184 , Kawai et al. $^{2}$ report measurements of the afterglow of GRB 050904 at optical wavelengths. And, on page 181 , Haislip et al. ${ }^{3}$ detail the tightly choreographed sequence of observations necessary to detect the barely visible afterglow at even longer, near-infrared wavelengths.

In astronomy, distance, time and the wavelength at which observations are made are inextricably linked. Light travels at a finite speed, and so takes a finite time to get to us. Far-off objects are thus seen as they were in the past. Cosmologists generally use the redshift $z$ to designate distance or, equivalently, lookback time. The quantity $1+z$ is the factor by which the Universe has expanded between the time when a source emitted the light we observe and the present, and is also the factor by which the wavelength of light reaching us has been stretched owing to the expansion of the Universe.

So by measuring the wavelengths of absorption lines in the spectrum of the optical afterglow of GRB 050904, and comparing these with wavelengths characteristic of the heavier elements such as carbon, sulphur and silicon that are found in stars, the redshift of GRB 050904 was found ${ }^{2}$ to be $z=6.295$. It is thus the most distant stellar explosion ever witnessed, and occurred when the Universe was about $7 \%$ of its current age. Setting a new record for the farthest stellar explosion might seem noteworthy enough, but distance alone does not make GRB 050904 interesting. Its attraction lies in what it can reveal about star formation early in the history of the Universe.

Over the past decade, astronomers have been particularly successful in tracing how early stellar systems changed and coalesced to become the 'respectable' elliptical, spiral and irregular galaxies seen in the present-day Universe. These early galaxies are far less luminous than quasars, but are still (as we see them) forming new stars, and so emitting copious amounts of light. Among these, the galaxy hosting the youngest-known quasar ${ }^{4}$ gives the earliest example of a star-forming region ${ }^{6}$. The presence in this galaxy of a large amount of mass in the form of interstellar molecules and dust is proof not only that substantial star formation was taking place only 800 million years after the Big Bang, but also that the process of chemical enrichment - in which heavier elements synthesized by previous generations of stars are incorporated into the galaxy - was well advanced.

Star formation in these early epochs was therefore probably not too different from that taking place now in some regions of the local Universe. It is in itself no surprise that a GRB can occur at these early times: GRBs are thought to be produced by the deaths of a rare breed of massive stars ${ }^{7}$, and, the more massive a star, the shorter its lifetime. Stars slightly more massive than our Sun have lifetimes that are shorter than the age of the Universe at $z=6.295$, and a star 20 times as massive can keep going for only a thousandth as long.

So far, however, GRB 050904 is the only example of a stellar explosion that occurred at a time in the evolution of the Universe before 'reionization', the point when the hydrogen between galaxies was completely ionized by ultraviolet radiation. Neutral hydrogen in the Universe at $z=6.295$ would have been a very effective absorber of any photon from GRB 050904 emitted at a wavelength shorter than the hydrogen 'Lyman $\alpha$ ' line at $121.6 \mathrm{~nm}$. Interestingly, such a damped Lyman $\alpha$ absorption profile of neutral hydrogen is not easily discernible in the optical afterglow spectrum ${ }^{2}$ of GRB 050904. Instead, one sees flux absorbed by the presence of a dense screen of metalenriched gas in the immediate surroundings, presumably ejected by the massive stellar progenitor prior to collapse. This might make GRBs less suited as clean markers of when, exactly, the reionization epoch occurred ${ }^{8}$. More optimistically, the detection of metal absorption lines in the afterglow spectrum from the gas around the burst site offers an unusual opportunity to study the physical conditions in the interstellar medium at these early epochs.

GRB observations will certainly pave the way for the discovery of more primitive objects than the galaxies presently known at $z=6.5$. Distant galaxies are themselves difficult to study because they appear faint and small. GRBs will serve not only as signposts to such galaxies, but could be used to study the gradual build-up of heavy elements in them to determine the conversion history of primordial gas into stars. Although the light from GRB 050904 is likely to have come from a 


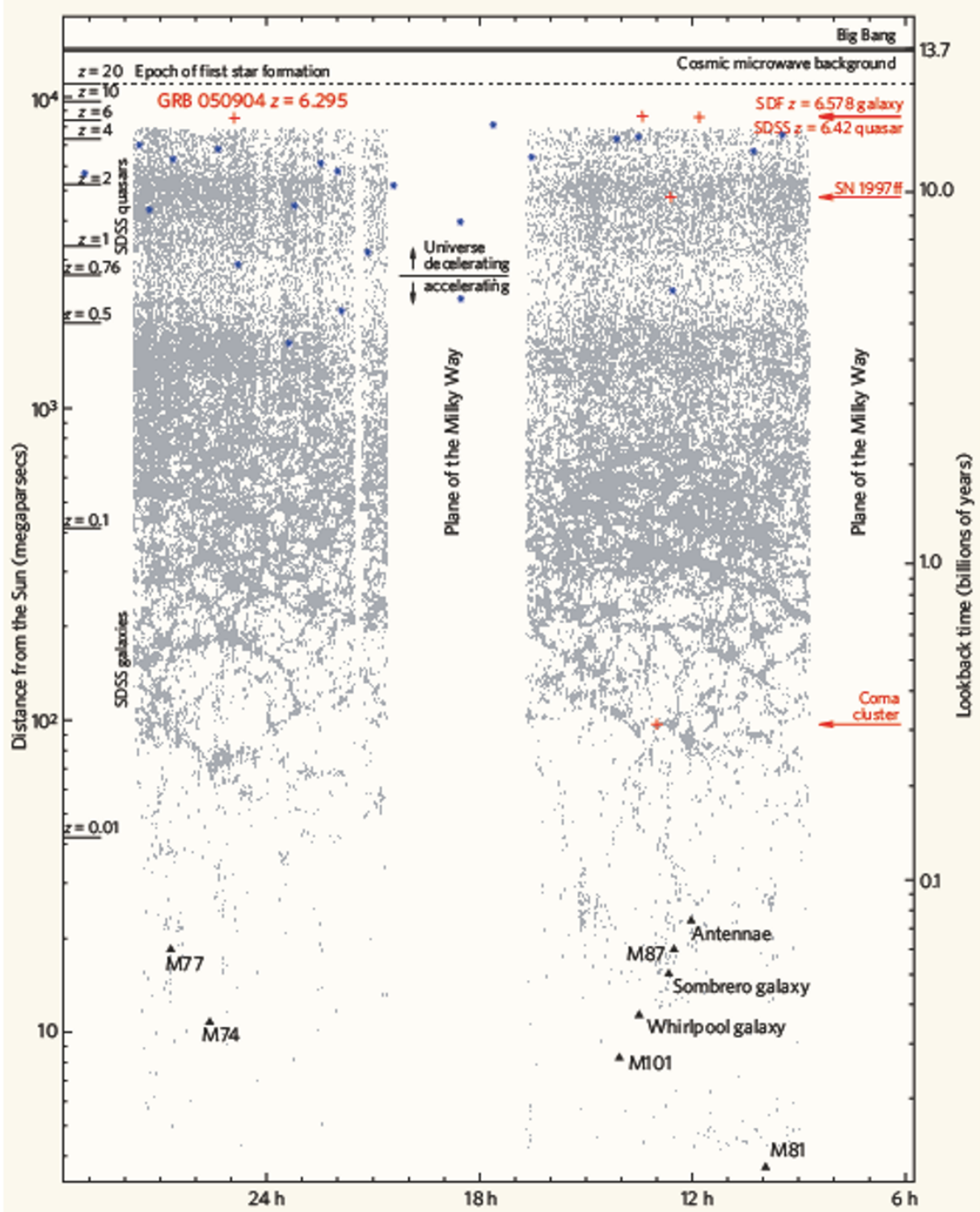

Figure 1 | The Universe as we know it. A $360^{\circ}$ vista showing the entire sky, with visible structures stretching backin distance, time and redshift. (Theimmediate light and dust from our own Galaxy, the Milky Way, obscures all light from outside sources in its plane.) The most distant light we observe comes from the radiation left over from the Big Bang: the cosmic microwave background. As we descend the chart, we find the most distant objects known, followed by a web of Sloan Digital Sky Survey (SDSS) quasars and galaxies. Closer to home, we start to see a collection of familiar 'near' galaxies (triangles). At $z=0.7554$ (in the favoured cosmological models), the Universe undergoes a transition from a slowing expansion to an accelerating one. Objects with $z<0.7554$ are observed at an epoch when the Universe's expansion is accelerating, whereas objects farther away are observed at an epoch when the Universe's expansion is decelerating. Also marked are all Swift GRBs with known distances (blue stars); SN 1997ff, the most distant type Ia supernova at $z=1.7$; and the archetypal large galaxy cluster, the Coma cluster. The most distant GRB, reported in this issue ${ }^{1-3}$, is at redshift $z=6.295$, comparable to the most distant galaxies found by the Subaru Deep Field (SDF) survey, and the most distant SDSS quasar. (Courtesy of J. R. Gott and M. Juric ${ }^{10}$.)

later star that was already metal-enriched, bursts arising from the very first generation of stars ${ }^{9}$ (those comprising only light nuclei such as hydrogen and helium produced in the Big Bang) may be detectable. And if bursts can be detected from a time before galaxies had gravitationally assembled, they might even provide a glimpse into the pregalactic phase of the Universe. Enrico Ramirez-Ruiz is at the Institute for Advanced Study, Einstein Drive,
Princeton, New Jersey 08540, USA. e-mail: enrico@ias.edu

1. Cusumana G etal. Nature 440, 164 (2006).

2. Kawai, N.et al. Nature 440, $184-186$ (2006).

3. Haislip, I B.etal. Nature 440, 181-183(2006).

4. Fan, X etal. Astion J. 125, 1649-1699 (2008).

5. Kodaira, K.etal. Publ. Astion Soc. Jpn 55, L17-L21 (200B).

6. Walter, F. etal. Nature 424, 406-408(2003)

7. Hjorth, Let al. Nature 423,847-850 (2003).

8. Barkana, R\& Loeb, A. Astraphys J. 601, 64-77(2004)

9. Abel, T. etal. Science 295,93-98 (2002).

10. Gott, J.R et al. Astrophys. J. 624,463-484 (2005)

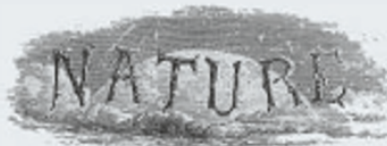

\section{YEARS AGO}

Ageing in Industry by F. Le Gros

Clark and Agnes C. Dunne - The only people who seem to have solved the problem of their senescent members seem to have beenthe mythical Hyperboreans whose aged, when they wished to retire from life, performed a "joyful suicide" by decking themselves with garlands and precipitating themselves from a rock into the sea. Non-mythical peoples have found various other solutions. The Tibarini used to hang their old on gibbets, the Hircanians and Bactrians cast them, while still living, to the dogs, and the Scythians buried them alive. In Great Britain, such drastic measures have never been contemplated, although the plight of the aged has often given cause for much distress. During the past two or three decades we have become increasingly conscious of the wider repercussions of our ageing population and the resulting social and economic disadvantages. From Nature 10 March 1956.

\section{YEARS AGO}

Geschichte der biologischen Theorien, seit dem Ende des siebzehnten Jahrhunderts by Dr. Em. Radl - Althoughbiology is now permeated by the evolution idea, and has continually before it the ideal of giving a genetic description of the present phase of the animate world, there is some reason to fear, as Dr. Radl indicates, a growing apathy towards the study of the evolution of the science itself. Whether itbe that many workers share Nietzsche's view that the study of history paralyses the intelligence, or that they feelit their primary business to make history, not to read it, or that they regard historical inquiries as the philosopher's task... The modern biologist, intent on new discoveries, hasno use for Aristotle, Descartes and Leibnitz, but their influence may be upon him none the less... even the most modern system of biology is, like our own body, a museum of relics. From Nature 8March 1906.

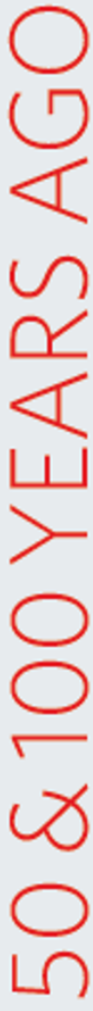

